Elsevier required licence: (C) <2019>. This manuscript version is made available under the CC-BY-NCND 4.0 license http://creativecommons.org/licenses/by-nc-nd/4.0/

The definitive publisher version is available online at

[https://www.clinical-lymphoma-myeloma-leukemia.com/article/S2152-2650(19)31430-2/fulltext] 


\section{Accumulation of CD69+ Terminal Effector CD8+ $T$ cells occurs in the bone marrow of newly diagnosed Myeloma patients who lack protective clonal $\mathrm{Vb}$ expanded cytotoxic $\mathrm{T}$ cells}

Christian Bryant, ${ }^{1}$ Ka Hei Aleks Lau, ${ }^{2}$ Slavica Vuckovic, ${ }^{1}$ Felix Marsh-Wakefield, ${ }^{3}$ Annabel Kruzins, ${ }^{4}$ Helen McGuire, ${ }^{5}$ Shihong Yang, ${ }^{6}$ Barbara Fazekas de St. Groth, ${ }^{5}$ Najah Nassif, ${ }^{7}$ Scott N. Byrne, ${ }^{0}$ John Gibson, ${ }^{2}$ Christina Brown, ${ }^{6}$ Stephen Larsen, ${ }^{6}$ Derek McCulloch, ${ }^{6}$ Richard Boyle, ${ }^{6}$ Douglas Joshua, ${ }^{9}$ P. Joy $\mathrm{Ho}^{10}$

${ }^{I}$ Royal Prince Alfred Hospital, Sydney, NSW $;{ }^{2}$ Royal Prince Alfred Hospital, Camperdown, NSW; ${ }^{3}$ The University of Sydney, N/A; ${ }^{4}$ School of Life Sciences, University of Technology Sydney, N/A; ${ }^{5}$ Ramaciotti Facility for Human Systems Biology, The University of Sydney, N/A; ${ }^{6}$ Royal Prince Alfred Hospital, N/A; ${ }^{7}$ School of Life Sciences, University of Technology Sydney, Sydney, NSW; ${ }^{8}$ The University of Sydney, Discipline of Infectious Diseases and Immunology, N/A; ${ }^{9}$ Faculty of Health Science, University of Sydney, Sydney, NSW; ${ }^{10}$ Royal Prince Alfred Hospital, Camperdown, New South Wales, Australia.

The investigation of the differentiation of terminal effectorCD8+CD57+T cells (TTE) in multiple myeloma (MM) and its premalignant stage, monoclonal gammopathy of undetermined significance (MGUS), is essential for understanding myeloma immune escape. We have previously shown that patients with clonal expansions of cytotoxic TTE cells have better outcomes than those who do not, but have not examined the underlining differentiation of TTE cells that leads to these distinct states. We hypothesised that a lack of clonally expanded TTE cells in myeloma patients could be due to myeloma induced alterations of TTE cell differentiation. To define TTE cell differentiation at high resolution, we have analysed paired bone marrow (BM) and peripheral blood (PB) samples from patients with newly diagnosed Myeloma (NDMM) and MGUS using time of flight mass cytometry and unsupervised clustering algorithm Flow Self-organizing Map (FlowSOM). We designed a 39 antibody panel, including antibodies to TCR-Vb families custom-labelled with heavy metals, allowing the detailed characterization of the phenotype of clonally expanded TTE cells. We found that in contrast to TTE cells in MGUS, TTE cells in BM of NDMM failed to display the phenotype typical of terminal effector cell differentiation and were enriched in atypical tissue resident CD69+, CD28+ and CD27+ subsets. They also failed to display the expected upregulation ofCD45RA and downregulation of CD45RO expression. Moreover, the accumulation of atypical CD69+ TTE cells was prevalent in myeloma patients without clonally expanded TTE cells. FlowSOM clustering discovered 4 metaclusters (MC) contributing to the accumulation of CD69+ TTE cells in the BM of NDMM patients. Phenotypically, two MC were in the memory stage based on CD27, CD28 and CD45RO expression and another two MC showed evidence of progression to the effector stage by downregulating CD27, CD28, CD45RO and upregulating CD45RA expression. Progression to the effector stage was associated with downregulation of CD38 and PD-1 expression, but did not affect persistent TIGIT expression. Vb expanded TTE cells did not contribute to the accumulation of CD69+ TTE cells and occupied phenotypically different MC with low or undetectable CD69 expression. When comparing phenotype of $\mathrm{Vb}$ expanded TTE cells to remaining TTE cells, effector and memory CD8+T cells they had higher Tbet, Perforin, Granzyme B, and lower levels of Eomes, TIGIT and PD-1 expression arguing against their exhaustion stage and confirming our previous observations that they resemble senescent cells. Our results suggest that accumulation of atypical tissue resident CD69+ TTE cells in myeloma infiltrated BM could prevent differentiation and expansion of clonal myeloma specific CD8+TTE cells and ultimately contribute to myeloma immune escape. 